\title{
A model of low-velocity impact damage assessment of laminated composite structures
}

\author{
Konstantinos P. Stamoulis ${ }^{1, *}$, Stylianos K. Georgantzinos ${ }^{2}$, and Georgios I. Giannopoulos ${ }^{3}$ \\ ${ }^{1}$ Aviation Academy, Faculty of Technology, Amsterdam University of Applied Sciences, Amsterdam, \\ Netherlands \\ ${ }^{2}$ Machine Design Laboratory, Department of Mechanical Engineering and Aeronautics, University of \\ Patras, Patras, Greece \\ ${ }^{3}$ Department of Materials Science, University of Patras, Patras, Greece
}

\begin{abstract}
Laminated composites have important applications in modern aeronautical structures due to their extraordinary mechanical and environmental behaviour. Nevertheless, aircraft composite structures are highly vulnerable to impact damage, either by low-velocity sources during maintenance or high-velocity sources during in-flight events. Even barely visible impact damage induced by low-velocity loading, substantially reduces the residual mechanical performance and the safe-service life of the composites structures. Despite the extensive research already carried out, impact damage of laminated composite structures is still not well understood and it is an area of on-going research. Numerical modelling is considered as the most efficient tool as compared to the expensive and time-consuming experimental testing. In this paper, a finite element model based on explicit dynamics formulations is adopted. Hashin criterion is applied to predict the intra-laminar damage initiation and evolution. The numerical analysis is performed using the ABAQUS ${ }^{\circledR}$ programme. The employed modelling approach is validated using numerical results found in the literature and the presented results show an acceptable correlation to the available literature data. It is demonstrated that the presented model is able to capture forcetime response as well as damage evolution map for a range of impact energies.
\end{abstract}

\section{Introduction}

Laminated composites are increasingly being used in the aviation industry due to their superior mechanical properties (specific stiffness and strength) and their competitive, through-life environmental behaviour (fatigue and corrosion resistance). These characteristics make them preferred materials for lightweight and more efficient aerospace structures, all of which translates into reduced costs [1]. However, it is well-known that aircraft composite structures are susceptible to impact damage, either by low-velocity sources during maintenance (e.g. accidental collision with ground vehicles, dropped tool events) or high-velocity sources during in-flight events (e.g. bird or lighting strikes). The National Aerospace Laboratory (NLR) in the Netherlands investigated the amount of ground handling

*Corresponding author: k.stamoulis@hva.nl 
incidents in 2008 and found out that 1 out of 5000 flights had a ground handling event, ranging from a small scratch to severe damage. This means that approximately $60 \%$ of all aircraft incidents occur in combination with ground handling [2]. When subjected to lowvelocity impact loading, a composite structure is degraded through various failure mechanisms and may lose up to $50 \%$ of its strength even with a disregarded, barely visible impact damage (BVID) [3]. Despite extensive research carried out in the last two decades, impact damage of laminated composite structures is not well understood due to the complexity and non-linearity of the damage zone and it is still an area of on-going research [4]. Therefore, it is essential to study the low-velocity impact behaviour and model the damage development of composite laminates. Numerical modelling is considered as the most efficient tool as compared to the expensive and time-consuming experimental testing.

Numerous studies have been carried out to investigate the low velocity impact response of laminated composite structures. A reference list and a summary of some important earlier numerical studies is reported in a recent paper [5]. Furthermore, an extensive literature review for contact and impact dynamics is provided by Gilardi and Sharf [6]. In this paper, a finite element approach is adopted for the low-velocity impact simulations of the laminated composite structure that accounts for intralaminar damage (matrix and fibre-dominated damage). The proposed method is based on explicit dynamics formulations and also employs the Hashin criterion [7] in order to predict the intra-laminar damage initiation and evolution. The numerical analysis was performed using the ABAQUS ${ }^{\circledR}$ programme [8]. The simulation results show good agreement with corresponding data found in the open literature, where possible. The numerical analysis demonstrates that the model can reasonably capture both the qualitative and quantitative aspects of intra-laminar damage (matrix and fibre-dominated damage) for a range of impact energies. Specifically, the presented computational approach is able to predict the force-time response generated due to the impact which is considered as the most important parameter influencing the structural integrity of the structure. Furthermore, it can capture damage parameter maps showing the damage shape evolution in the matrix material or fibre, and thus can be used as a damage assessment computational tool.

\section{Computational approach}

In order to perform an accurate assessment of the structural integrity of a component after a low-velocity impact, an efficient computational tool predicting its response under the specific impact conditions could be very valuable. Based on this concept, the proposed method concerning aeronautical structures is based on explicit dynamics formulations. The computational approach is described in the following sections.

\subsection{Geometry}

The impactor is assumed to be a rigid ball of $20 \mathrm{~mm}$ diameter with an initial velocity specified in a direction normal to the plane of the plate. The plate is a square of $80 \mathrm{~mm}$ side length. For efficiency reasons, the plate is modelled as a $2 \mathrm{D}$ structure via the utilization of conventional shell elements. Various values of plate thickness are adopted depending on the simulation test.

\subsection{Finite element model formulation}

The governing equations for an explicit or implicit dynamics problem may be expressed in matrix form as

$$
\mathbf{M} \ddot{\mathbf{u}}(t)+\mathbf{K u}(t)=\mathbf{F}(t)
$$


where $\mathbf{M}$ and $\mathbf{K}$ are the mass and stiffness matrices of dimensions equal to the number of degrees of freedom, $\mathbf{u}$ is the generalized nodal coordinates vector, and $\mathbf{F}(t)$ is the force vector due to the impact. The contact force is obtained by using the penalty contact algorithm [8]. The mass and stiffness matrices may be obtained by using conventional finite element assembly techniques according to the geometric, material and mesh characteristics. Concerning explicit dynamics analysis and for a state of dynamic equilibrium, the constructing diagonal (lumped) element mass matrices are used in order to compute the nodal accelerations $\ddot{\mathbf{u}}$ at the beginning of each time increment according to the following equation:

$$
\ddot{\mathbf{u}}_{(i)}=\mathbf{M}^{-1}\left(\mathbf{F}_{(i)}-\mathbf{F}_{\text {in }(i)}\right)
$$

where $\mathbf{F}_{\text {in }(i)}$ is the internal force vector, and subscript $i$ refers to the time increment number within an explicit dynamic step. The explicit solver [8] integrates nodal accelerations that are calculated at the time point $t$ using the explicit central-difference integration algorithm to advance the velocity solution $\dot{u}$ to time $t+\Delta t / 2$ and the displacement solution $u$ to time $t+\Delta t$ as:

$$
\begin{gathered}
\dot{u}_{\left(i+\frac{1}{2}\right)}=\dot{u}_{\left(i-\frac{1}{2}\right)}+\frac{\Delta t_{(i+1)}+\Delta t_{(i)}}{2} \ddot{u}_{(i)} \\
u_{(i+1)}=u_{(i)}+\Delta t_{(i+1)} \dot{u}_{\left(i+\frac{1}{2}\right)}
\end{gathered}
$$

where $u$ is a degree of freedom (displacement or rotation). The central-difference integration algorithm is explicit in a sense that kinematic state is advanced using the known values of $\dot{u}_{\left(i-\frac{1}{2}\right)}$ and $\ddot{\mathrm{u}}_{(i)}$ from the previous time step. The explicit solver can perform analysis on a large number of small inexpensive time increments to ensure that the central-difference integration rule is stable. In order to extract accurately the dynamics response in such a ratesensitive problem, the higher time increment size $(\Delta t)$ is estimated as $\Delta t=\sqrt{\rho / E} L^{e}$ where $\rho$ is the material density, $E$ is the modulus of elasticity, and $L^{e}$ is the smallest element length.

\subsection{Progressive damage model for composite structures}

Damage initiation and propagation in composite laminated structures is far more complex than that of isotropic materials. In composites, each layer is subject to damage or failure; therefore, a single layer or a part of it may suffer damage that propagates within the same layer or even to other layers. When the damage propagates within the laminate, the load is redistributed within each layer according to the stiffness degradation, so the damage progression does not necessarily indicate complete failure of the structure. To simulate this phenomenon, Hashin's failure criterion is implemented. According to Hashin theory [7], the damage initiation should be activated by meeting one of the following failure criteria:

Fiber tension:

$$
\left(\frac{\widehat{\sigma}_{11}}{X^{\mathrm{T}}}\right)^{2}+\gamma\left(\frac{\widehat{\sigma}_{12}}{S^{\mathrm{L}}}\right)^{2}=1,0 \leq \gamma \leq 1
$$


Matrix tension:

$$
\left(\frac{\widehat{\sigma}_{22}}{Y^{\mathrm{T}}}\right)^{2}+\left(\frac{\widehat{\sigma}_{12}}{S^{\mathrm{L}}}\right)^{2}=1
$$

Fiber compression:

$$
\left(\frac{\widehat{\sigma}_{11}}{X^{\mathrm{C}}}\right)^{2}=1
$$

Matrix compression:

$$
\left(\frac{\widehat{\sigma}_{22}}{2 S^{\mathrm{T}}}\right)^{2}+\left[\left(\frac{Y^{\mathrm{C}}}{2 S^{\mathrm{T}}}\right)^{2}-1\right] \frac{\widehat{\sigma}_{22}}{Y^{\mathrm{C}}}+\left(\frac{\widehat{\sigma}_{12}}{S^{\mathrm{L}}}\right)^{2}=1
$$

where $X^{\mathrm{T}}$ is the tensile strength in the fiber direction, $X^{\mathrm{C}}$ is the compressive strength in the fiber direction, $Y^{\mathrm{T}}$ is the tensile strength in the direction perpendicular to the fibers, $Y^{\mathrm{C}}$ is the compression strength in the direction perpendicular to the fibers, $S^{\mathrm{L}}$ and $S^{\mathrm{T}}$ are the longitudinal and transverse shear strengths, respectively, $\gamma$ is a coefficient that determines the contribution of the shear stress to the fiber tensile initiation criterion, and $\hat{\sigma}$ is the effective stress which is introduced to account for the area reduction and is defined as:

$$
\hat{\sigma}=\left[\begin{array}{ccc}
\frac{1}{1-d_{f}} & 0 & 0 \\
0 & \frac{1}{1-d_{m}} & 0 \\
0 & 0 & \frac{1}{1-d_{s}}
\end{array}\right] \sigma
$$

where $d_{f}, d_{m}$, and $d_{s}$ are damage variables associated with fiber, matrix, and shear damage, respectively, while $\sigma$ is the apparent stress prior to damage initiation. These damage variables can have values between zero and one, depending on the damage progression.

\section{Results and discussion}

The demonstration of the method accuracy is required in order to be considered capable for damage assessment in real aeronautical structures. For this reason, aluminium and composite materials are used and impact conditions similar to those found in the open literature are assumed for validation purposes. The complete set of tests performed in this work and the corresponding physical properties and conditions are presented in Table 1.

The first set of tests (Test 1 ) concerns a typical aluminium plate. The elastic mechanical properties for the specific isotropic material are $E=70 \mathrm{GPa}$ (Young's modulus) and $v=0.3$ (Poisson's ratio). In the first transition impact simulation, the plate is considered to behave fully elastically. The contact force that is generated due to the impact is considered as the most important parameter influencing the structural integrity of the structure. For this reason, it is calculated and its time-history is depicted in Fig. 1. 
Table 1. Physical Properties of the tested models.

\begin{tabular}{|c|l|c|c|}
\hline Test & & Impactor & Plate \\
\hline \multirow{4}{*}{1} & Geometry & Radius $=10 \mathrm{~mm}$ & $80 \times 80 \times 5 \mathrm{~mm}^{3}$ \\
\cline { 2 - 4 } & Material model & Rigid & Aluminum, elastic \& bilinear plastic \\
\cline { 2 - 4 } & Inertia properties & $m_{i}=30 \mathrm{gr}$ & $\rho=2500 \mathrm{~kg} / \mathrm{m}^{3}$ \\
\cline { 2 - 4 } & Initial \& boundary conditions & $v_{0}=10 \mathrm{~m} / \mathrm{s}$ & Clamped - clamped \\
\hline \multirow{3}{*}{2} & Geometry & Radius $=10 \mathrm{~mm}$ & $80 \times 80 \times 2 \mathrm{~mm}^{3}$ \\
\cline { 2 - 4 } & Material model & Rigid & Composite, Hashin damage \\
\cline { 2 - 4 } & Inertia properties & $m_{i}=10,20,30 \mathrm{gr}$ & $\rho=1620 \mathrm{~kg} / \mathrm{m}^{3}$ \\
\cline { 2 - 4 } & Initial \& boundary conditions & $v_{0}=10 \mathrm{~m} / \mathrm{s}$ & Simply supported \\
\hline
\end{tabular}

Since the magnitude of contact force takes relatively high values despite the low energy of the impact, the plastic behavior should be also considered. Hence, a bi-linear plastic model for the aluminum is also assumed in the next simulation. The yield stress is considered to be $S_{y}=200 \mathrm{GPa}$. The corresponding contact force is calculated and presented in Fig. 1, as well. It is noticed that there is a remarkable difference in the contact force response and, thus, the plastic response of a ductile material should be introduced in such a computational model. The maximum contact force calculated in the elastic case is $8229 \mathrm{~N}$ and the corresponding one in the bi-linear model is $6948 \mathrm{~N}$.

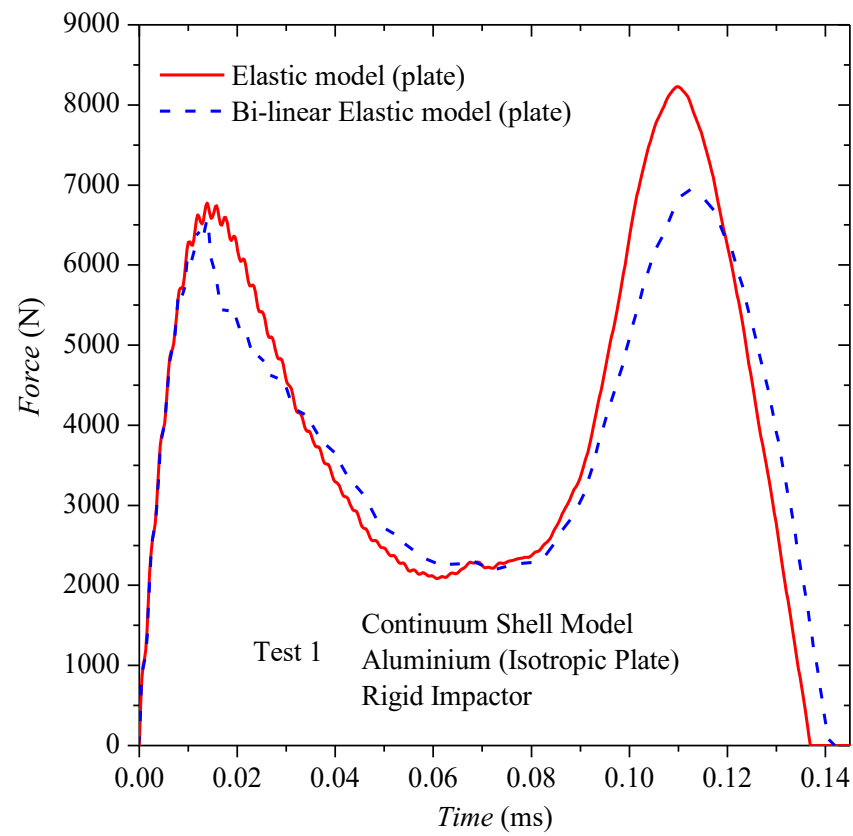

Fig. 1. Contact force - time history under low velocity transition impact for rigid impactor and aluminium plate.

The next set of tests (Test 2) concerns laminated composite materials. The physical properties and impact test boundary and initial conditions are described in Table 1. The orthotropic material parameters as well as the values of the progressive damage model parameters are presented in Table 2 . 
Table 2. Mechanical properties of composite structures used in different tests.

\begin{tabular}{|c|c|}
\hline Lamina Properties & Test 2 \\
\hline$E_{11}(\mathrm{GPa})$ & 153 \\
\hline$E_{22}(\mathrm{GPa})$ & 10.3 \\
\hline$G_{12}(\mathrm{GPa})$ & 5.2 \\
\hline$v_{12}$ & 0.3 \\
\hline Hashin damage parameters & \\
\hline$X^{T}(\mathrm{GPa})$ & 2.1 \\
\hline$X^{C}(\mathrm{GPa})$ & 1.1 \\
\hline$Y^{T}(\mathrm{GPa})$ & 0.135 \\
\hline$Y^{C}(\mathrm{GPa})$ & 0.21 \\
\hline$S^{T}(\mathrm{GPa})$ & 0.07 \\
\hline Fiber Direction & {$[0 /-45 / 45 / 90 / 0 /-45 /-45 / 90] \mathrm{s}$} \\
\hline
\end{tabular}

The finite element analysis reveals the contact force time-history behavior that is illustrated in Fig. 2. In order to examine the effect of impact energy in damage occurred after a low velocity impact, different values of the impactor mass are assumed. The higher the impactor mass, the higher the contact force during the transition impact.

Here, as also in Fig. 1, multiple peaks of contact force are depicted. This phenomenon is also observed in a previous relevant work [4] concerning the transition impact simulation. This could be explained from the vibrational behaviour of the plate during the impact, in which the fundamental and higher modes of plate vibrations force the plate contact area to move in the same or the opposite direction with respect to the impactor velocity.

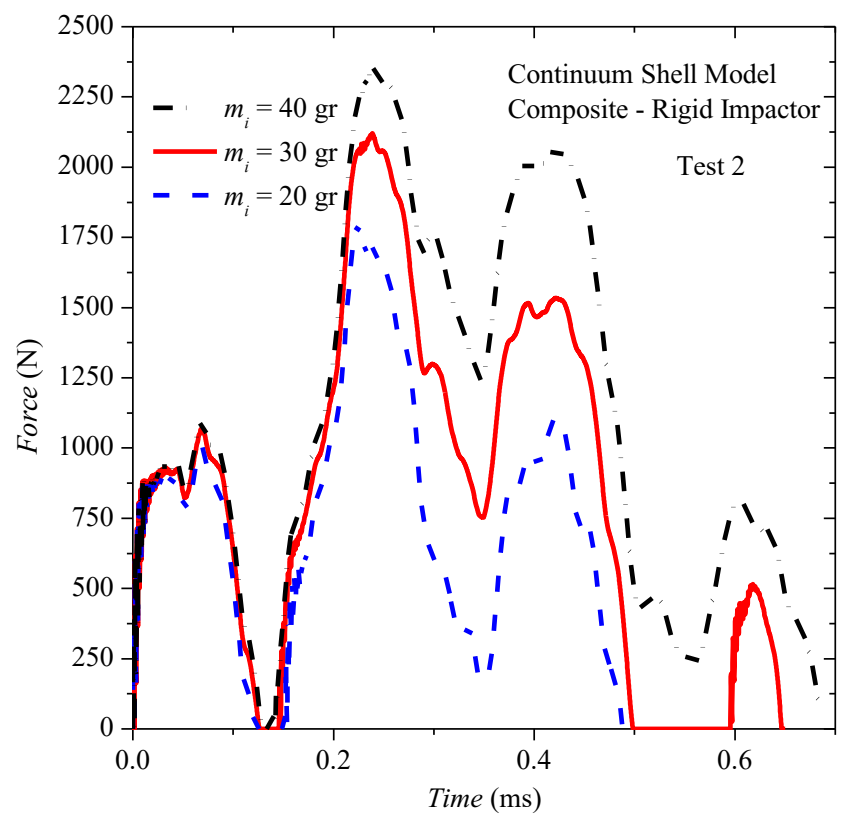

Fig. 2. Contact force - time history under low velocity impact for rigid impactor and composite plate. 
The calculated values of maximum contact force in all simulations are compared with the corresponding ones extracted by other methods, where possible. The comparison which is provided in Table 3, reveals a reasonable agreement between the different methods. Since the developed computational models are now validated, they can be utilized for a damage assessment of laminated composite structures suffered from a low-velocity impact.

Table 3. Maximum contact force $(\mathrm{N})$. Comparisons with numerical results found in the open literature.

\begin{tabular}{|c|c|c|c|c|}
\hline Test & Present & Solid [4] & Continuum Shell [4] & External contact law [4] \\
\hline 1 & 8229 (Elastic) & 7028 & 8052 & 6863 \\
& 6948 (Bi-linear) & & & 2240 \\
\hline 2 & 2120 & - & 1846 & \multicolumn{2}{c}{} \\
\hline
\end{tabular}

Fig. 3 depicts the matrix damage which occurs in two plies of the composite structure due to various low-velocity impacts. The damaged area described by the red color, while the undamaged one by the blue. It is noticed that no damage is predicted for the fibres regarding the specific impact energy cases. As expected, the damage is higher as the impact energy increases due to the higher impactor mass. The shape of the damage area illustrated in damage parameter maps are consistent with the corresponding ones found in the literature $[5,9]$.

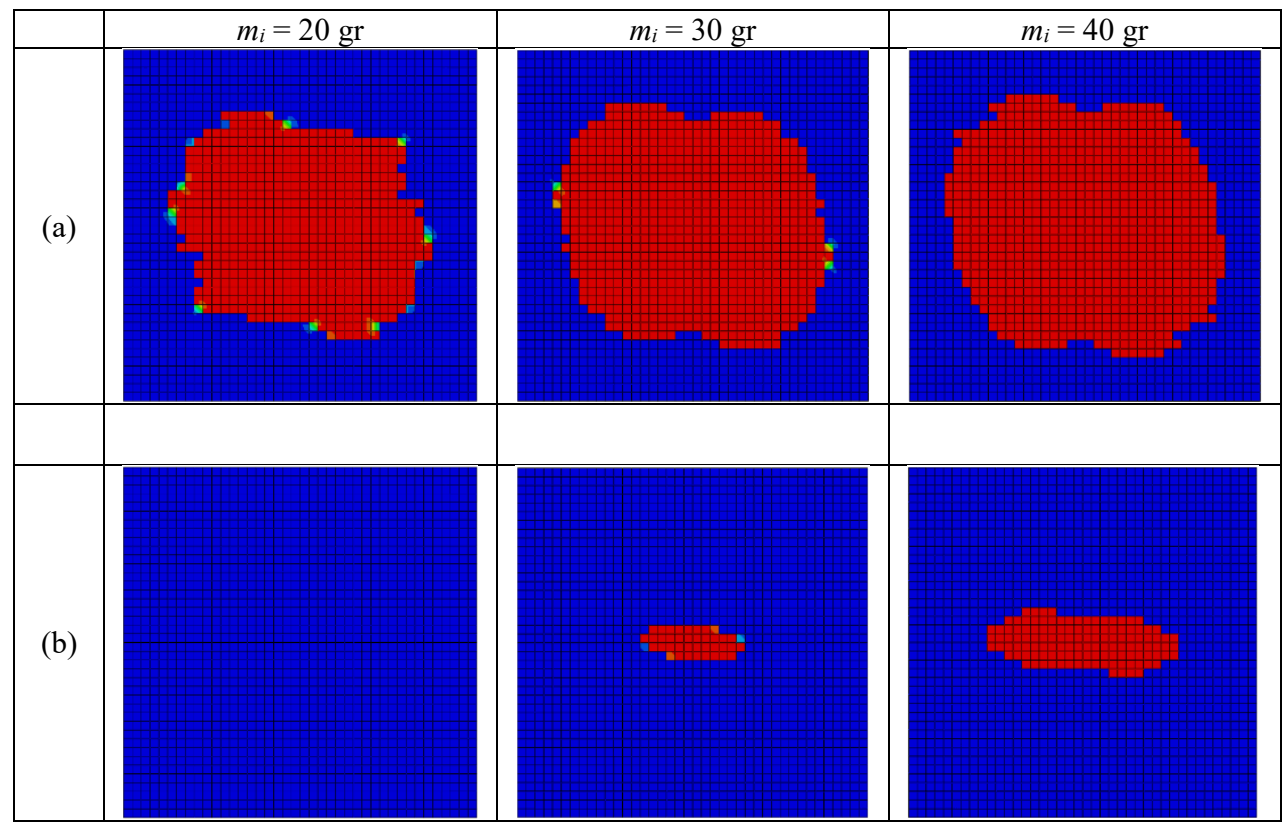

Fig. 3. Matrix damage assessment in a composite structure after a low-velocity impact event. The red and blue colour depict the damaged and undamaged area, respectively, for the case (a) ply $0^{\circ}$ (front), and (b) ply ply $0^{\circ}$ (symmetric) and for various impact energies $\left(v_{0}=10 \mathrm{~m} / \mathrm{s}\right)$. 


\section{Conclusions}

A composite damage model that accounts for intralaminar damage (matrix and fibredominated damage) has been presented. The comparisons with results taken from the literature have shown a high degree of capability in predicting impact characteristics, as well as impact damage. According to the results of this work, the following concluding remarks arise:

- The non-elastic behaviour of the material influences remarkably the results of a low velocity impact simulation and, thus, it is required to be incorporated into the computational model.

- The model is able to capture force-time response for a range of impact energies.

- The contact force and the damage area increase as the impact energy becomes higher.

- The method is capable of capturing damage parameter maps showing the damage shape in matrix material or fibre, which can be used as a damage assessment computational tool.

\section{References}

1. M. C. Y. Niu, Composite Airframe Structures - Practical Design Information and Data (Conmilit Press Ltd., Hong Kong, 1992)

2. AviationFacts.eu, Damage Detection in Composites in Aviation (Amsterdam University of Applied Sciences, Amsterdam, 2015)

3. M.O.W. Richardson, M.J. Wisheart, Compos. A. 27, 1123-31 (1996)

4. A.H.A. Ibrahim, A.S. Yigit, ASME. J. Comput. Nonlinear Dyn. 10, 061009 (2015)

5. C. Zhang, E. A. Duodu, J. Gu, Compos. Struct. 173, 219-27 (2017)

6. G. Gilardi, I. Sharf, Mech. Mach. Theory 37, 1213-39 (2002)

7. Z. Hashin, J. Appl. Mech. 47, 329-34 (1980)

8. Abaqus Documentation, (Dassault Systèms, 2014)

9. P. Rozylo, H. Debski, T. Kubiak, Compos. Struct. 181, 158-170 (2017) 